PREPARED FOR THE U.S. DEPARTMENT OF ENERGY, UNDER CONTRACT DE-AC02-76CH03073

PPPL-3639

PPPL-3639

UC-70

A Model of Solar Flares Based on Arcade Field Reconnection and Merging of Magnetic Islands

by

G.S. Choe and C.Z. Cheng

December 2001

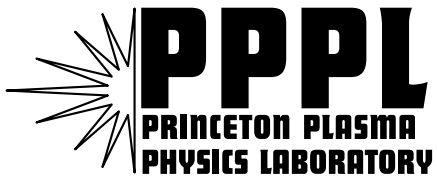

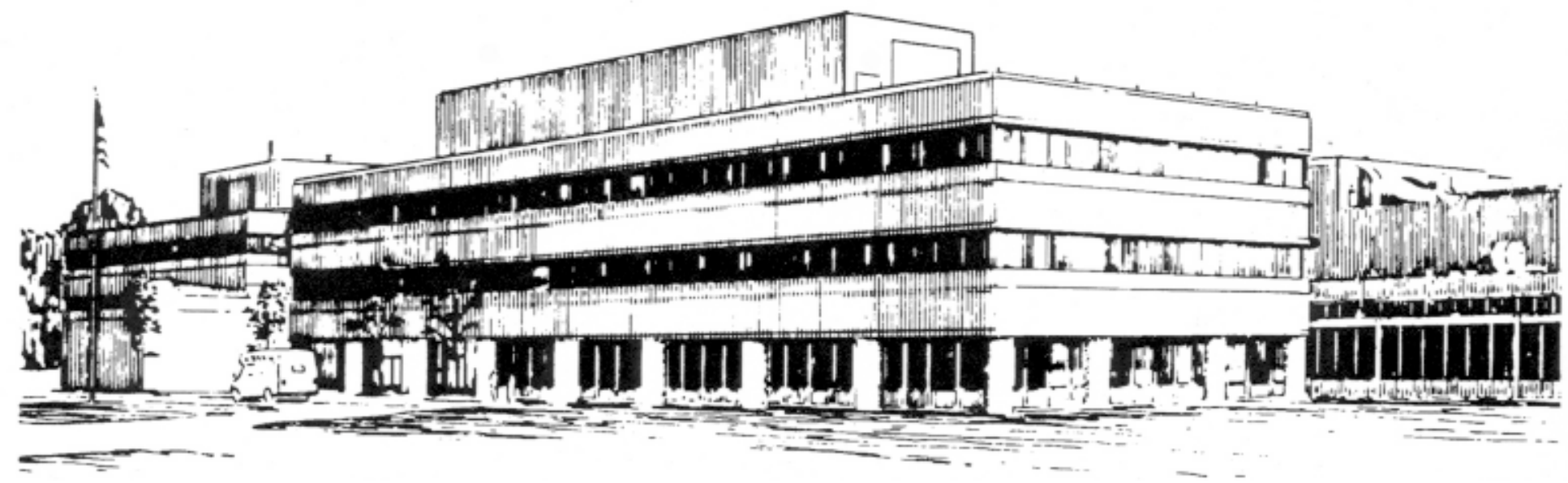

PRINCETON PLASMA PHYSICS LABORATORY PRINCETON UNIVERSITY, PRINCETON, NEW JERSEY 


\section{PPPL Reports Disclaimer}

This report was prepared as an account of work sponsored by an agency of the United States Government. Neither the United States Government nor any agency thereof, nor any of their employees, makes any warranty, express or implied, or assumes any legal liability or responsibility for the accuracy, completeness, or usefulness of any information, apparatus, product, or process disclosed, or represents that its use would not infringe privately owned rights. Reference herein to any specific commercial product, process, or service by trade name, trademark, manufacturer, or otherwise, does not necessarily constitute or imply its endorsement, recommendation, or favoring by the United States Government or any agency thereof. The views and opinions of authors expressed herein do not necessarily state or reflect those of the United States Government or any agency thereof.

\section{Availability}

This report is posted on the U.S. Department of Energy's Princeton Plasma Physics Laboratory Publications and Reports web site in Fiscal Year 2002. The home page for PPPL Reports and Publications is: http://www.pppl.gov/pub_report/

DOE and DOE Contractors can obtain copies of this report from:

U.S. Department of Energy

Office of Scientific and Technical Information

DOE Technical Information Services (DTIS)

P.O. Box 62

Oak Ridge, TN 37831

Telephone: (865) 576-8401

Fax: (865) 576-5728

Email: reports@adonis.osti.gov

This report is available to the general public from:

National Technical Information Service

U.S. Department of Commerce

5285 Port Royal Road

Springfield, VA 22161

Telephone: 1-800-553-6847 or

(703) 605-6000

Fax: (703) 321-8547

Internet: http://www.ntis.gov/ordering.htm 
To appear in Physics of Plasmas

\title{
A model of solar flares based on arcade field reconnection and merging of magnetic islands
}

\author{
G. S. Choe and C. Z. Cheng \\ Princeton Plasma Physics Laboratory, Princeton University, Princeton, NJ 08543-0451
}

(November 14, 2001)

\begin{abstract}
Solar flares are intense, abrupt release of energy in the solar corona. In the impulsive phase of a flare, the intensity of hard X-ray emission reaches a sharp peak indicating the highest reconnection rate. It is often observed that an Xray emitting plasma ejecta (plasmoid) is launched before the impulsive phase and accelerated throughout the phase. Thus, the plasmoid ejection may not be an effect of fast magnetic reconnection as conventionally assumed, but a cause of fast reconnection. Based on resistive magnetohydrodynamic simulations, a solar flare model is presented, which can explain these observational characteristics of flares. In the model, merging of a newly generated magnetic island and a pre-existing island results in stretching and thinning of a current sheet, in which fast magnetic reconnection is induced. Recurrence of homologous flares naturally arises in this model. Mechanisms of magnetic island formation are also discussed.
\end{abstract}

Typeset using REVTEX 


\section{INTRODUCTION}

Solar flares are observed in the solar corona and chromosphere as a brilliant flash of light (electromagnetic waves) in various wavelengths. A large flare can release over $10^{32}$ erg of energy in an hour or so. By emission characteristics, the evolution of a flare can be divided into several phases. ${ }^{1,2}$ The preflare phase, which lasts about 10 minutes before the flare onset, is characterized by enhanced thermal emissions from the coronal plasma mostly in soft X-rays $(h \nu<10 \mathrm{keV})$. Then, the intensity and area of the emission in all wavelengths (dominantly in soft $\mathrm{X}$-rays and $\mathrm{H}_{\alpha}$ ) rapidly increase for about 5 minutes in the flash phase. During this phase, hard X-rays $(h \nu>10 \mathrm{keV})$ and microwave bursts are often observed for 10-100 seconds and this duration is called the impulsive phase. Then, in the main phase, overall emission intensity slowly declines over about an hour or sometimes as long as a day.

It is generally believed that the energy released during a flare is a part of the magnetic free energy that has been stored in stressed magnetic fields and that magnetic reconnection plays a central role in tapping the free energy. ${ }^{3}$ There have been a lot of flare models, which propose diverse magnetic field configurations possibly leading to flare magnetic reconnection. ${ }^{4-9}$ Among these, flare models resorting to magnetic reconnection in bipolar magnetic fields are collectively referred to as CSHKP (Carmichael-Sturrock-Hirayama-Kopp-Pneuman) ${ }^{6-9}$ models ${ }^{10}$ although the global field geometries, especially above the reconnection site, and the proposed mechanisms of flare trigger are all different. Later numerical models with bipolar arcades ${ }^{11-14}$ are also grouped into CSHKP type models. Some observations by Xray telescopes onboard Yohkoh have provided a strong support for CSHKP models. The observation of a hard X-ray (HXR) source above the soft X-ray (SXR) loop ${ }^{15}$ has confirmed a reconnection site above the loop. The morphology of SXR emissions in the shape of a candlelight (or a cusp) ${ }^{16}$ indicates magnetic reconnection occurring in CSHKP type field configurations.

In simulations of CSHKP type models, ${ }^{11^{-14}}$ a magnetic island (also called a plasmoid) is formed by magnetic reconnection of arcade field lines and the island is accelerated while 
reconnection is active. However, recent observations of plasmoid ejection do not support the idea that the plasmoid is entirely driven by magnetic reconnection underneath. Yohkoh observations show that most flares are indeed associated with X-ray plasma ejecta (or plasmoids), but some ejecta are launched well before the flare impulsive phase. ${ }^{17}$ Observations have also revealed that ejecta in X-ray flares undergo a sharp increase in their rising speed before the main peak of the hard X-ray emission and are further accelerated throughout the impulsive phase. ${ }^{18,19}$ It is thus inferred that the plasmoid ejection is not a consequence of the flare, but a cause of it. Based on these observations, Shibata ${ }^{20}$ proposed a plasmoid-inducedreconnection model, in which a fast reconnection responsible for a flare is triggered by the plasmoid ejection. However, the formation and acceleration mechanism of the plasmoid was left unaddressed in the model.

It is often observed that solar flares take place repetitively in the same active region with essentially the same position and with a common pattern of development. Such flaring phenomena are called homologous flares. The time interval between successive flaring events varies from several hours to a few days. ${ }^{21}$ Woodgate $^{22}$ suggested that a majority of flares might be homologous in the sense that the footpoints reappear very near the same place. Thus, it was even argued that flare homology must be included among the constraints on flare models. ${ }^{23}$ In spite of the importance of the homologous recurrence of flares, there have been few studies of it. Repetition of solar eruptions was studied by Linker and Mikić2 using resistive magnetohydrodynamic (MHD) simulations, but little connection was found between individual eruptive events.

The observational conditions for homologous flares are not different from general conditions for flares. ${ }^{25}$ Among those, the presence of magnetic shear along the polarity inversion line is a necessary condition, and the reformation of filaments (prominences) after a flare is also notable. Since prominence material is believed to reside at the bottom of helical field lines ${ }^{26}$ helical flux ropes are regarded to play a role in flares. An increase of magnetic shear means an increase of magnetic helicity. Magnetic helicity can be injected into the corona either by shearing (twisting) footpoint motions in the photosphere or by emergence 
of twisted fields. Our study deals with a magnetic field system into which magnetic helicity is continuously injected. We study the resistive evolution of a coronal arcade system under continuous footpoint motions and investigate the generation and interaction of magnetic islands and magnetic reconnection inducing and induced by those processes. In the following section, our modeling procedure is described. Using the numerical results, the flare mechanism is expounded in Sec. III. Sec. IV presents another possible mechanism of magnetic island formation different from that in Sec. III. A brief summary with a discussion follows in Sec. V.

\section{DESCRIPTION OF MODELING}

In this study, we investigate the evolution of bipolar magnetic arcades in a Cartesian coordinate system. The polarity inversion line is taken to lie on the $z$-axis, in which direction all physical quantities are assumed to be invariant. The coordinate in the vertical direction is designated as $y$. Our model is two and half-dimensional in the sense that all the variables depend on $x$ and $y$ only while vector quantities have all three components. The magnetic field is thus expressed as

$$
\mathbf{B}(x, y)=\hat{\mathbf{z}} \times \nabla \psi(x, y)+B_{z}(x, y) \hat{\mathbf{z}}
$$

It is supposed that the magnetic field is potential (current-free) at $t=0$ and then evolves in response to the horizontal plasma flows in the solar surface for $t>0$. If a shearing boundary flow $V_{z}(x, t)=v_{z}(x, y=0, t)$ is imposed, the displacement in the $z$-direction of the fluid element at the boundary location $x$ is

$$
\zeta(x, t)=\int_{0}^{t} V_{z}\left(x, t^{\prime}\right) d t^{\prime}
$$

On the other hand, the distance in the $z$-direction between the two conjugate footpoints of a field line labeled by $\psi$ is given by

$$
\Delta Z(\psi)=Z\left(x^{+}[\psi]\right)-Z\left(x^{-}[\psi]\right)=\int_{\psi} \frac{B_{z}}{|\nabla \psi|} d s_{p},
$$


where $Z(x)$ is the $z$-coordinate of the footpoint at $x$ of the field line labeled by $\psi$. The above integration is performed along the field line designated by $\psi$ and $s_{p}$ is the arclength of the field line projected on the $x y$-plane. If the plasma occupying the whole space is a perfect conductor and if there is no reconnection, the distance in the $z$-direction between two conjugate field line footpoints is the same as the sum of the magnitudes of the plasma displacement at $x^{+}$and at $x^{-}$, i.e.,

$$
\Delta Z(\psi)=\Delta \zeta(\psi)=\zeta\left(x^{+}\right)-\zeta\left(x^{-}\right)
$$

The toroidal flux ( $B_{z}$ flux) through the area made by the unit poloidal flux around the field line labeled by $\psi$, which will be called the differential $B_{z}$ flux at $\psi$, is

$$
\phi_{z}(\psi)=\int_{\psi} \frac{B_{z}}{|\nabla \psi|} d s_{p}
$$

Therefore,

$$
\phi_{z}(\psi)=\Delta Z(\psi)
$$

which tells that the distance in the $z$-direction between two conjugate footpoints is nothing but the differential $B_{z}$ flux.

When there exists diffusion of magnetic fields, the differential $B_{z}$ flux is not conserved even if the plasma at the boundary does not move. Thus, Eq. (4) does not hold under magnetic diffusion. The discrepancy between $\Delta Z(\psi)$ and $\Delta \zeta(\psi)$ is negligible in a system with a very small resistivity such as the solar atmosphere unless there is magnetic reconnection. When magnetic reconnection is allowed in a magnetic arcade, a magnetic island can be created by reconnection and there can be more than one flux surface labeled by $\psi$. In this case,

$$
\phi_{z}(\psi)=\sum_{i} \phi_{z i}(\psi)=\sum_{i} \Delta Z_{i}(\psi)=\sum_{i} \int_{\psi} \frac{B_{z}}{|\nabla \psi|} d s_{p i}
$$

where the index $i$ designates the different flux surface labeled by the same $\psi$. In other words, the total differential $B_{z}$ flux at $\psi$ is the sum of the footpoint distance in the $z$ direction for the field line $\psi$ connected to the boundary and the pitch of the helical field 
line $\psi$ in a magnetic island for one rotation in the poloidal plane. Ideally, we may think of pointwise magnetic reconnection with a diffusion region of a zero volume. In this pointwise reconnection process, the toroidal flux contained in the entire flux volume between $\psi$ and $\psi+d \psi$ are conserved. Only in this ideal case, it holds that

$$
\Delta \zeta(\psi)=\sum_{i} \Delta Z_{i}(\psi)=\phi_{z}(\psi)
$$

In reality, a reconnection region has a finite volume however small it may be and $\phi_{z}(\psi)$ is not conserved through reconnection and even diverges at the separatrices connected to the X-line. Away from the separatrix, however, Eq. (8) holds approximately in a small resistivity system. ${ }^{13}$ This implies that a reconnection process redistributes the differential $B_{z}$ flux, which was contained in an arcade flux tube, into two different flux volumes: one in a new arcade and the other in a magnetic island. In our simulation, we impose velocity vectors at the boundary as boundary conditions and have control of $\zeta(\psi)$ rather than $Z(\psi)$, which comes out from the solution. The maximum plasma displacement at the boundary at time $t$ will be denoted by $\zeta_{m}(t)$.

The equations governing the evolution of our model corona are a full set of $2 \frac{1}{2} \mathrm{D}$ MHD equations including gravity and resistivity as follows:

$$
\begin{gathered}
\frac{\partial \rho}{\partial t}+\nabla \cdot(\rho \mathbf{v})=0 \\
\rho\left(\frac{\partial \mathbf{v}}{\partial t}+\mathbf{v} \cdot \nabla \mathbf{v}\right)=\mathbf{J} \times \mathbf{B}-\nabla p+\rho \mathbf{g}+\nabla \cdot \mu \nabla \mathbf{v} \\
\frac{\partial \psi}{\partial t}+\mathbf{v} \cdot \nabla \psi=\eta \nabla^{2} \psi \\
\frac{\partial B_{z}}{\partial t}=\frac{\partial}{\partial x}\left(v_{z} B_{x}-v_{x} B_{z}\right)+\frac{\partial}{\partial y}\left(v_{z} B_{y}-v_{y} B_{z}\right) \\
+\nabla \cdot\left(\eta \nabla B_{z}\right) \\
\frac{d}{d t}\left(\frac{p}{\rho^{\gamma}}\right)=0
\end{gathered}
$$




$$
\begin{gathered}
p=\rho R T \\
B_{x}=-\frac{\partial \psi}{\partial y}, \quad B_{y}=\frac{\partial \psi}{\partial x}, \\
\mathbf{J}=\nabla \times \mathbf{B},
\end{gathered}
$$

where all the quantities are expressed in a nondimensionalized form by a proper normalization. The magnetic field $\mathbf{B}$ is normalized by $B_{0}=50 \mathrm{G}$ the maximum magnitude of the boundary normal field, the mass density $\rho$ by the initial density $\rho_{0}=\left(1.0 \times 10^{9} \mathrm{~cm}^{-3}\right) m_{\mathrm{H}}$ at the bottom boundary, the velocity $\mathbf{v}$ by $v_{0}=B_{0} /\left(4 \pi \rho_{0}\right)^{1 / 2} \approx 3.2 \times 10^{3} \mathrm{~km} \mathrm{~s}^{-1}$, the time $t$ by $t_{0}=L_{0} / v_{0} \approx 9.3 \mathrm{~s}$, and the resistivity $\eta$ by $L_{0} v_{0}$, in which $L_{0}=3.0 \times 10^{4} \mathrm{~km}$ is the length unit. The nondimensionalized resistivity is approximately the inverse of the Lundquist number. To treat the energetics of the solar corona properly, we need to consider the coronal heating, radiative cooling and anisotropic heat conduction. ${ }^{27}$ However, without a well established knowledge in the coronal heating mechanism, we adopt a polytropic relation [Eq. (13)] instead of a full energy equation. Even with this simplification, it is not easy to determine the polytropic index $\gamma$ representing the entire coronal plasma. Considering the high thermal conductivity in the corona, we just set $\gamma=1$ in Eq. (13) assuming an isothermal atmosphere with temperature of $2 \times 10^{6} \mathrm{~K}$. Although this setting does not allow our model to accommodate the flare heating in a self-contained manner, the overall dynamics is not much affected by this simplification because the plasma $\beta$ is very low. ${ }^{28}$

The photosphere modeled by the bottom boundary is considered as a rigid, perfect conductor. All the other boundaries are assumed to be open outward so that waves generated in the computational domain can freely go out. The governing equations are finite-differenced and integrated in time employing a semi-implicit scheme. ${ }^{29,27}$ For a detailed account of the modeling procedures, readers are referred to Refs. 30 and 31. 


\section{FLARING MECHANISM}

In our simulation study, we impose a shearing footpoint motion at the bottom boundary to inject magnetic helicity into the system and induce reconnection of arcade fields creating a magnetic island. The maximum shearing speed $V_{z 0}$ is set $10^{-3} v_{0}$ lest that the system should be driven away from equilibrium. Although simulations are performed with different values of resistivity, all the simulation runs show similar evolutionary trends. As the magnetic shear is increased by the shearing footpoint motion, the current layer in the center of the magnetic arcade becomes thinner and longer so that magnetic reconnection takes place and a magnetic island is created. This result was already reported by previous numerical simulation studies. ${ }^{12^{-14}}$ With $\eta=10^{-5}$, the magnetic reconnection is found to be triggered around $t \approx 11000 t_{0}$ which corresponds to $\zeta_{m} \approx 11$. The arcade field reconnection divides and redistributes the toroidal flux that was previously contained in line-tied nested flux tubes into two new flux systems: one with the magnetic island and the other with the linetied reconnected field. The magnetic shear in the arcade under the magnetic island is thus reduced because the reconnected field lines in the underlying arcade have a smaller conjugate footpoint distance in the $z$-direction than the old field lines before magnetic reconnection occurs. By a continuing shearing motion, the magnetic shear in the underlying arcade is again increased and a new reconnection is found to be initiated at $t \approx 19000 t_{0}\left(\zeta_{m} \approx 19\right)$. The new magnetic island so created rises and pushes up the line-tied field surrounding it so that this line-tied field starts to reconnect with the field in the upper island through the upper X-line. Fig. 1 shows a 3D view of field lines including the upper magnetic island, the newborn island and three types of line-tied arcade fields in this stage. After all the linetied field in the underlying arcade surrounding the newborn island has reconnected with the upper island field, the two magnetic islands quickly coalesce to form one island. This merged island continues to rise gaining flux as field lines surrounding it continue to reconnect in the vertical current sheet below. The magnetic reconnection generating a new island, the newborn island's merging with an upper island and the reconnection under the integrated 
island are all interrelated and constitute a flaring event. Flaring events are repeated with some time interval while the shearing footpoint motion continues. This repetitive occurrence of a sequence of reconnection processes is interpreted as a set of homologous flares. In the case with $\eta=10^{-5}$, the time interval between successive flaring events is around $10000 t_{0}$, which is about a day.

The height of O-lines in the islands is shown in Fig. 2 as a function of time. Compared with the first island and other integrated islands, newborn islands rise far faster. For example, the rising speed of the integrated island formed at $t \approx 20000 t_{0}$ is about $1.3 \times 10^{-3} v_{0}$ $\left(\sim 4 \mathrm{~km} \mathrm{~s}^{-1}\right)$. On the other hand, the new island born at $t \approx 29000 t_{0}$ rises in the beginning at a speed of $\sim 3 \times 10^{-3} v_{0}\left(\sim 10 \mathrm{~km} \mathrm{~s}^{-1}\right)$ and in the merging stage at a speed of $\sim 2 \times 10^{-2} v_{0}$ $\left(\sim 65 \mathrm{~km} \mathrm{~s}^{-1}\right)$. With the shift in island generation, the integrated island as well as the newborn island gets faster. From other simulation runs with different resistivity values, we have found that the rising speed of islands also depends on the reconnection rate. With $\eta=5 \times 10^{-5}$, the rising speeds of newborn islands are more than twice of the speeds with $\eta=10^{-5}$. With $\eta=10^{-3}$ the rising speeds are almost ten times those with $\eta=10^{-5}$. The rising speed of a newborn island in a merging process is roughly proportional to $\eta^{1 / 2}$. From the above results, it should be noted that the motion of newborn islands comprises two phases; in the first phase, the island rises rather slowly, although faster than the pre-existing one, and in the second phase, it rises much faster. The first phase mainly involves an ideal MHD process leading to field compression and current sheet thinning above the newborn island. Dynamics in the second phase is mostly governed by magnetic reconnection between the upper island and the underlying flux system containing the newborn island. The rising speed of the newborn island thus depends on the reconnection rate. As can be seen in Fig. 2, a single island does not have the second phase with a faster motion. Although the altitude of the integrated island becomes higher and higher with time, it has moved only a few solar radii in a few days in our simulation.

To investigate our simulation results in the aspects of the energy generation and dissipation, we show in Fig. 3 the evolution of (a) the ratio of the total magnetic energy to the 
potential field energy, $W_{\mathrm{B}} / W_{\text {pot }}$, (b) the energy input through the bottom boundary per unit plasma displacement at $x= \pm L_{0}$, which is equivalent to the upward Poynting flux through the bottom boundary normalized with the maximum shearing speed $V_{z 0}$, and (c) the maximum magnitude of the $z$-component of current density, $\left|J_{z}\right|_{\max }$, in the vertically elongated current layer. The upward Poynting flux through the bottom boundary is calculated as

$$
\int_{-\infty}^{\infty} S_{y}(x, y=0) d x=-\int_{-\infty}^{\infty} B_{y} B_{z} v_{z} d x .
$$

In Fig. 3, results from four simulation runs with different values of resistivity $\left(\eta=0,2 \times 10^{-6}\right.$, $10^{-5}$ and $5 \times 10^{-5}$ ) are displayed. In our simulations, the energy release by reconnection processes is an increasing function of resistivity. The time interval between the initiation of a new reconnection marked with a filled circle in the figure and the completion of island merging marked with a blank circle is a decreasing function of resistivity. These facts just imply that the reconnection rate in our simulation system is an increasing function of resistivity. In MHD simulations employing a spatially uniform resistivity, it is well known that the reconnection rate is an increasing function of resistivity. ${ }^{32}$ This may not be true in the real solar plasma, in which the diffusion region has a much smaller volume ${ }^{13}$ and microscale physics plays a more dominant role ${ }^{33}$ than in MHD simulations like ours. Thus, the purpose of our running cases with different resistivity values is to find the effect of different reconnection rates, not that of resistivity values.

Now we investigate the relationship between different reconnection processes and the observed phases of solar flares. The evolution of magnetic energy (Fig. 3a) evidently shows that the magnetic energy released by merging of two islands is smaller than the energy released by the subsequent reconnection of line-tied field under the integrated island although the former process proceeds much faster than the latter. Thus, the main phase of a flare can be attributed to the reconnection in a vertically elongated current sheet under the integrated island as in conventional pictures of solar flares. ${ }^{34,9,35}$ Fig. $3 \mathrm{c}$ shows the maximum current density in the vertically elongated current sheet, $\left|J_{z}\right|_{\max }$. Because the reconnection electric field, given by $E_{z, R e c}=\eta J_{z}$ in the current sheet, equals to the poloidal flux reconnected per 
unit time, the $\left|J_{z}\right|_{\max }$ curve indicates how much flux is being reconnected in the X-line. As shown in Fig. 3c, the maximum current density starts to increase before the initiation of reconnection in the underlying arcade, continues to increase during the new island formation, peaks at the time of island merging completion, and then slowly decays until a new current sheet is formed in the underlying arcade. The time interval from the reconnection trigger in the underlying arcade and to the end of the slow island rising phase can be interpreted as the preflare phase. The reconnection rate further increases in the fast island rising phase and reaches a maximum when merging of the two islands is completed. This rather short time interval is identified with the impulsive (or flash) phase. The highest reconnection rate in this phase is attributed to the shooting up of the lower island that elongates the line-tied field wrapping around both islands to form a very thin current sheet. After the merging of two islands, reconnection of line-tied field continues, but slows down with decreasing $\left|J_{z}\right|_{\text {max }}$. This phase is longer than the former two phases and is considered as the main phase of a flare. From Fig. 3c, we can obtain the reconnection electric field $E_{z, \text { Rec }}$. For $\eta=10^{-5}, E_{z, R e c} \approx 0.64 \mathrm{Vm}^{-1}$ at the time of the second island formation $\left(t \approx 1.9 \times 10^{4} t_{0}\right)$ when $\left|J_{z}\right|_{\text {max }} \approx 4.0 J_{0}$ and $E_{z, R e c} \approx 1.1 \mathrm{Vm}^{-1}$ at the completion of island merging when $\left|J_{z}\right|_{\text {max }} \approx 6.7 J_{0}$. With this electric field, electrons can be accelerated to an energy of $10 \mathrm{keV}$ in mere $10 \mathrm{~km}$. Although the classical resistivity in the actual solar corona is much lower $\left(\eta \sim 10^{-12}\right)$ than the resistivity values used in our simulation, the reconnection electric field required by the observed energy release in a flare (at least $\sim 10^{29} \mathrm{erg}$ in the impulsive phase of ten minutes or less) is not less than the value found in our simulation.

In Fig. 3, it is notable that the time interval between successive flaring events is a decreasing function of resistivity, or in other words, of reconnection rate. In Fig. 3b, we can also notice that the energy input rate has an exact anti-correlation with the magnetic energy. The energy input starts to increase when the magnetic energy starts to decrease by a new reconnection event and its maxima coincide with the magnetic energy minima. Also, the overall energy input rate is higher in cases with higher resistivity where magnetic reconnection is more active. Now we address the question how a magnetic reconnection process in 
the corona is related with the energy input through the solar surface. The upward Poynting vector is expressed as $S_{y}=-B_{z} v_{z} B_{y}$ [Eq. (17)] when only a shearing motion is imposed. Since $B_{y}(x, y=0)$ is constant in time and so is $v_{z}(x, y=0) / V_{z 0}$ except for the short initial ramp period, $S_{y} / V_{z 0}$ is most of time proportional to the toroidal magnetic field $B_{z}$. Thus, we have to examine the evolution of $B_{z}$ to understand the evolution of the Poynting flux. When arcade field lines are reconnected forming a magnetic island, the toroidal flux that was held in the line-tied arcade before the reconnection is redistributed into the magnetic island and the reconnected underlying arcade. The magnetic shear (more exactly the differential toroidal flux) is thus reduced in the reconnected line-tied field. Geometrically, the distance between two conjugate footpoints of the reconnected line-lied field line, $\Delta Z(\psi)$, is reduced compared with the footpoint distance of the field line before reconnection because the reconnection process cuts and joins two field line segments from different field lines in the same flux surface. A reduction of the differential toroidal flux $\Delta Z$, however, does not lead to a reduction of the toroidal field $B_{z}$ because the flux volume is also divided by the reconnection process and the flux volume of the reconnected line-tied field is smaller than before the reconnection. Therefore, we still do not have enough information to determine whether the toroidal field is increased or decreased by reconnection. In Fig. 3b, one can see that in the ideal MHD case, in which all the field lines are always tied to the bottom boundary, the energy input rate increases up to $\zeta_{m} \approx 2$ and then decreases. Thus, the toroidal magnetic field $B_{z}$ also first increases with the footpoint displacement up to a certain maximum and then asymptotically decreases in an ideal MHD system. The decrease of $B_{z}$ with increasing shear, which may sound strange, is a natural consequence of the fact that the total toroidal magnetic energy has an upperbound. ${ }^{36-38}$ Therefore, a reduction of $\Delta Z$ leads to an increase of $B_{z}$ as long as $\Delta Z$ is larger than a certain value $(\sim 4$ in our model). Moreover, the arcade under a new magnetic island is dynamically pressed by the reconnection outflows while reconnection is active. Therefore, the toroidal field is increased in the reconnected line-tied field and in consequence, the Poynting flux into the system is enhanced. This explains why the time interval between flaring events is shorter for a system 
with more active arcade reconnection.

\section{FORMATION OF MAGNETIC FLUX ROPES}

In Sec. III, we have treated a case that a magnetic island is formed by magnetic reconnection of line-tied arcade fields. However, our flare mechanism does not depend on the mechanism of magnetic island formation. We can think of other possibilities of appearance of magnetic islands (more exactly helical flux ropes) in the solar corona. First, a twisted helical flux rope may be formed below the solar surface and may emerge into the corona by magnetic buoyancy. ${ }^{39}$ Second, a magnetic flux rope can be created by magnetic reconnection between a pre-existing magnetic field and an emerging magnetic field, neither of which initially bears a helical structure. In this section, we examine the second possibility by a numerical simulation. As an initial field configuration, a moderately sheared bipolar arcade field is assumed. Near the center of this bipolar field, a new bipolar field is made to emerge from below the solar surface with opposite magnetic polarities to polarities of the pre-existing field. Since the poloidal fields of the two fields are in opposite directions, magnetic reconnection takes place if some resistivity is given. The reconnection creates two new line-tied arcade flux systems because the entire field system is now quadrupolar. When the emerging field has an axial field component in the opposite direction to that of the pre-existing field or when the emerging field has little axial field, the magnetic reconnection merely transfers fluxes from the pre-existing and emerging flux systems to the new arcade flux systems. In a $3 \mathrm{D}$ picture, this is the case that the pre-existing field and the emerging field subtend an angle close to $180^{\circ}$. What will happen if these two fields make an acute angle? This is the case that the axial components of both fields are parallel and at least one of them is strong enough. If magnetic reconnection takes place at one fixed point only, this case would not be different from the case with almost antiparallel fields. However, there is no reason for such a restriction on reconnection sites. Magnetic reconnection can take place at many different points, especially when the two fields make an an acute angle. In this 
case, a helical flux rope (a magnetic island in $2 \frac{1}{2} \mathrm{D}$ ) can be formed. ${ }^{40}$ As seen in Fig. 4 , a few magnetic islands are created in small scales in the current layer region, and they eventually merge to form one big, steady island. Magnetic reconnection thereafter just adds magnetic flux to this island. Our preliminary study shows that the subsequent evolution of the system can set free the island from the siege of the surrounding line-tied field. However, this is beyond the scope of the present paper.

\section{SUMMARY AND DISCUSSION}

The flare model presented in this paper can explain many observational features that could not be accommodated in previous flare models. Principal findings in our study are summarized in the following.

When a magnetic arcade is subject to a continuing shear-increasing footpoint motion, generation of magnetic islands via magnetic reconnection and their coalescence can repeatedly take place with some time interval. The series of these reconnection processes is regarded to constitute a sequence of homologous flares. The time interval between successive flaring events is shorter when the energy input rate into the system is higher.

The rising of a newborn island comprises a slower first phase and a faster second phase. The first phase involves arcade field reconnection creating a new island and is identified with the preflare phase. Coalescence of two magnetic islands takes place within a short time span in the second phase, which is identified with the impulsive (or flash) phase of a flare. The island merging process creates a long and thin current sheet below and facilitates reconnection of the line-tied field. The obtained reconnection electric field is large enough to accelerate electrons to an energy level higher than $10 \mathrm{keV}$ required for observed hard X-ray emissions. The arcade field reconnection under the integrated island persists for quite a long time after the island merging, although the reconnection electric field gradually decays. This phase, in which more energy is released in total than in the earlier two phases, is identified with the main phase of a flare. 
Our model can also be categorized into CSHKP models in the sense that it resorts to magnetic reconnection in bipolar magnetic arcades. However, our model is unique and distinct from the previous CSHKP models in that it can explain the observed rising motion of plasmoids and that it reverses the conventional cause-effect relationship between the arcade reconnection and the plasmoid ejection and acceleration. The homologous recurrence of flares is an intrinsic feature of our flare model. However, as discussed in our earlier paper, ${ }^{30}$ the time interval between flaring events highly depends on the pattern of shear-increasing motions. This explains why flares are not always observed as one of homologous flares.

Although we have created magnetic islands by arcade field reconnection in our simulations of flares, our flare model is quite independent of the origin of the magnetic islands. As another possible mechanism of magnetic island formation, we have shown that reconnection between magnetic fields subtending an acute angle can also create magnetic islands (helical flux ropes).

It is to be mentioned that the $2 \frac{1}{2} \mathrm{D}$ geometry has a few known disadvantages for realistic modeling of solar magnetic fields. First, the energy of even partially open fields is infinite in a slab parallel to the $x y$-plane and field line opening is thus energetically impossible. This is because the open field strength declines with radial distance like $1 / r .{ }^{41}$ The expulsion of a magnetic island to a long distance is also more difficult than in a spherical geometry. ${ }^{12,13}$ Second, a much larger footpoint displacement is required than in 3D models to create the same amount of shear angle, which is defined as the angle between the transverse component vector of the sheared field and that of the potential field. ${ }^{42}$ All these shortcomings rather help validate our model in a $2 \frac{1}{2} \mathrm{D}$ Cartesian geometry due to its restraining character; in other words, any violent behavior showing up in this model will take place in models with a more realistic geometry. 


\section{ACKNOWLEDGMENTS}

The authors sincerely thank the anonymous referee for the valuable comments. This work was supported by the DoE Contract DE-AC02-76-CHO3073 and the NSF grant ATM9906142. 


\section{REFERENCES}

${ }^{1}$ S. R. Kane, in Coronal Disturbances, IAU Symposium No. 5\%, edited by G. A. Newkirk (D. Reidel, Dordrecht, 1974), p. 105.

${ }^{2}$ E. R. Priest, Solar Magnetohydrodynamics (D. Reidel, Dordrecht, 1982).

${ }^{3}$ H. E. Petschek, in AAS-NASA Symposium on the Physics of Solar Flares, edited by W. N. Hess (NASA SP-50, Washington, DC, 1964), p. 425.

${ }^{4}$ P. A. Sweet, in Electromagnetic Phenomena in Cosmic Physics, edited by E. Lehnert (Cambridge University Press, Cambridge, 1958), p. 135.

${ }^{5}$ T. Gold and F. Hoyle, Mon. Not. R. Astron. Soc. 120, 89 (1960).

${ }^{6}$ H. Carmichael, in AAS-NASA Symposium on the Physics of Solar Flares, edited by W. N. Hess (NASA SP-50, Washington, DC, 1964), p. 451.

${ }^{7}$ P. A. Sturrock, Nature 211, 695 (1966).

8 T. Hirayama, Sol. Phys. 34, 323 (1974).

${ }^{9}$ R. A. Kopp and G. W. Pneuman, Sol. Phys. 50, 85 (1976).

${ }^{10}$ P. A. Sturrock, in Eruptive Solar Flares, IAU Colloquium 133, Lecture Notes in Physics, 399, edited by Z. Švestka, B. V. Jackson, and M. E. Machado (Springer-Verlag, Berlin, 1992), p. 397.

${ }^{11}$ Z. Mikić, D. C. Barnes, and D. D. Schnack, Astrophys. J. 328, 830 (1988).

12 Z. Mikić and J. A. Linker, Astrophys. J. 430, 898 (1994).

${ }^{13}$ G. S. Choe and L. C. Lee, Astrophys. J. 472, 372 (1996).

14 T. Amari, J. F. Luciani, J. J. Aly, and M. Tagger, Astron. Astrophys. 306, 913 (1996).

${ }^{15}$ S. Masuda, T. Kosugi, H. Hara et al., Nature 371, 495 (1994). 
${ }^{16}$ S. Tsuneta, Publ. Aston. Soc. Jpn. 47, 691 (1995).

${ }^{17}$ K. Shibata, S. Masuda, M. Shimojo et al., Astrophys. J. 451, L83 (1995).

${ }^{18}$ M. Ohyama and K. Shibata, Publ. Aston. Soc. Jpn. 49, 249 (1997).

${ }^{19}$ M. Ohyama and K. Shibata, Astrophys. J. 499, 934 (1998).

${ }^{20}$ K. Shibata, in Observational Plasma Astrophysics: Five Years of Yohkoh and Beyond, edited by T. Watanabe, T. Kosugi, and A. C. Sterling (Kluwer Academic Publishers, Boston, 1998), p. 187.

${ }^{21}$ M. Stix, The Sun: An Introduction (Springer-Verlag, Berlin, 1989).

${ }^{22}$ B. E. Woodgate, Adv. Space Res. 2, No. 11, 61 (1982).

${ }^{23}$ M. J. Hagyard, V. Gaizauskas, G. A. Chapman et al., in Energetic Phenomena on the Sun, edited by M. Kundu and B. E. Woodgate (NASA CP-2439, Washington, DC, 1986), p. 1.

${ }^{24}$ J. A. Linker and Z. Mikić, Astrophys. J. 438, L45 (1995).

${ }^{25}$ B. E. Woodgate, M.-J. Martres, J. B. Smith, Jr. et al., Adv. Space Res. 4, No. 7, 11 (1984).

${ }^{26}$ M. Kuperus and M. A. Raadu, Astron. Astrophys. 31, 189 (1974).

${ }^{27}$ G. S. Choe and L. C. Lee, Sol. Phys. 138, 291 (1992).

${ }^{28}$ L. C. Lee, Y. Lin, and G. S. Choe, Sol. Phys. 163, 335 (1996).

${ }^{29}$ D. S. Harned and D. D. Schnack, J. Comp. Phys. 65, 57 (1986).

${ }^{30}$ G. S. Choe and C. Z. Cheng, Astrophys. J. 541, 449 (2000).

${ }^{31}$ C. Z. Cheng and G. S. Choe, Earth, Planets and Space 53, 597 (2001).

${ }^{32}$ D. Biskamp, Nonlinear Magnetohydrodynamics (Cambridge University Press, Cambridge, 
1997).

${ }^{33}$ J. F. Drake, R. G. Kleva, and M. E. Mandt, Phys. Rev. Lett. 73, 1251 (1994).

${ }^{34}$ P. A. Sturrock, in Structure and Development of Solar Active Regions, IAU Symposium No. 35, edited by K. O. Kiepenheuer (D. Reidel, Dordrecht, 1968), p. 471.

${ }^{35}$ S. Tsuneta, Astrophys. J. 456, 840 (1996).

${ }^{36}$ J. J. Aly, Astrophys. J. 283, 349 (1984).

${ }^{37}$ J. J. Aly, Comput. Phys. Commun. 59, 13 (1990).

${ }^{38}$ G. S. Choe and L. C. Lee, Astrophys. J. 472, 360 (1996).

${ }^{39}$ E. N. Parker, Cosmical Magnetic Fields: Their Origin and Their Activity (Oxford University Press, Oxford, 1979).

${ }^{40}$ L. C. Lee and Z. F. Fu, J. Geophys. Res. 91, 6807 (1986).

${ }^{41}$ B. C. Low, Ann. Rev. Astron. Astrophys. 28, 491 (1990).

${ }^{42}$ S. K. Antiochos, R. B. Dahlburg, and J. A. Klimchuk, Astrophys. J. 420, L41 (1994). 


\section{FIGURES}

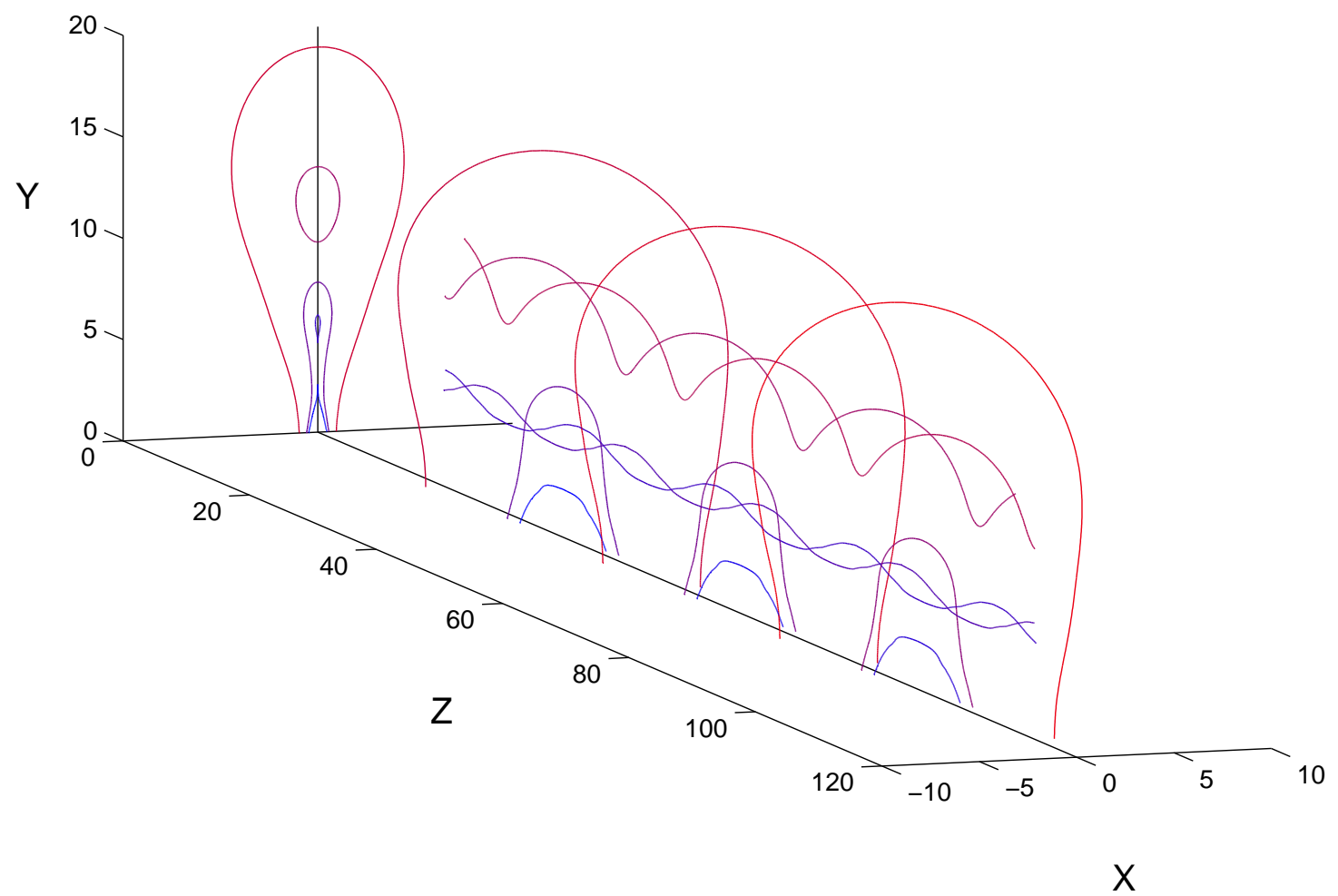

FIG. 1. A 3D view of field lines as well as their projection onto the $x y$-plane at an instance between the birth of a new magnetic island and the merging of two islands. The entire system consists of five flux systems: the upper pre-existing magnetic island, the lower newborn island, the line-tied field surrounding the upper island, the line-tied field surrounding the lower island, and the line-tied field under the lower island. 


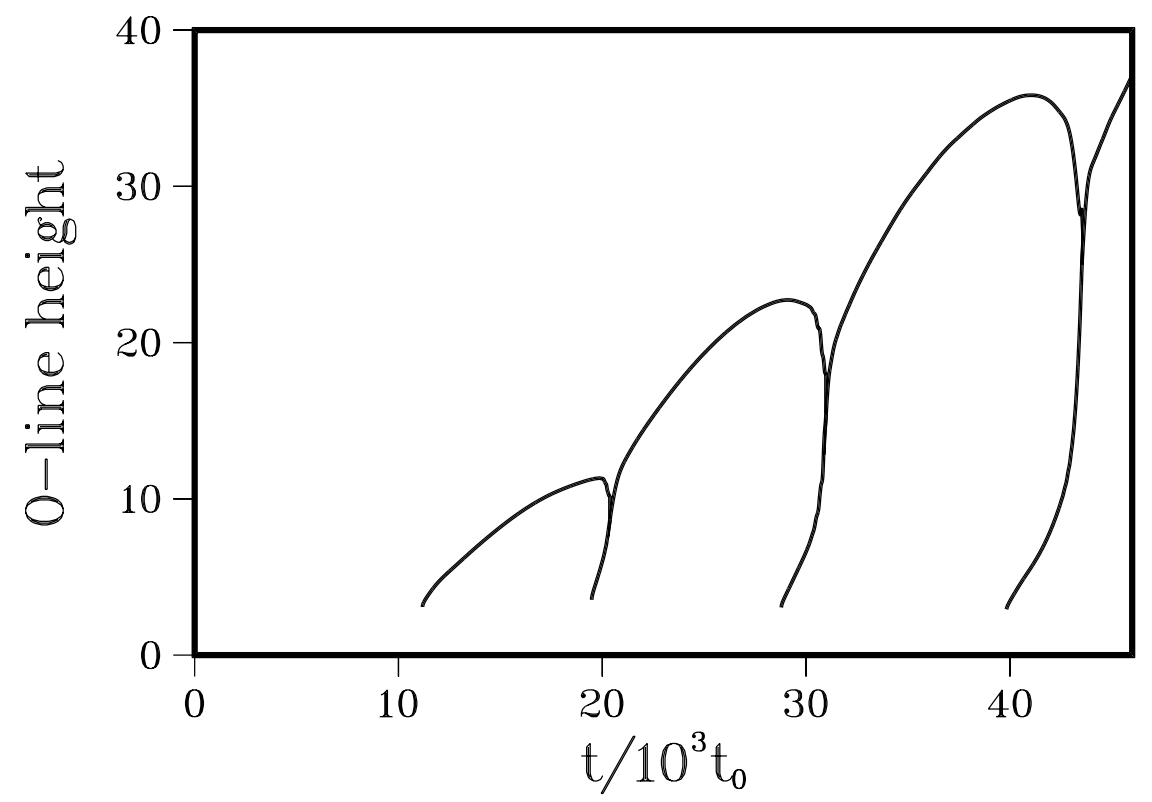

FIG. 2. The height of O-lines of the magnetic islands in the case with $\eta=10^{-5}$ as a function of time. The slope of the curves represents the rising speed of the magnetic islands. 

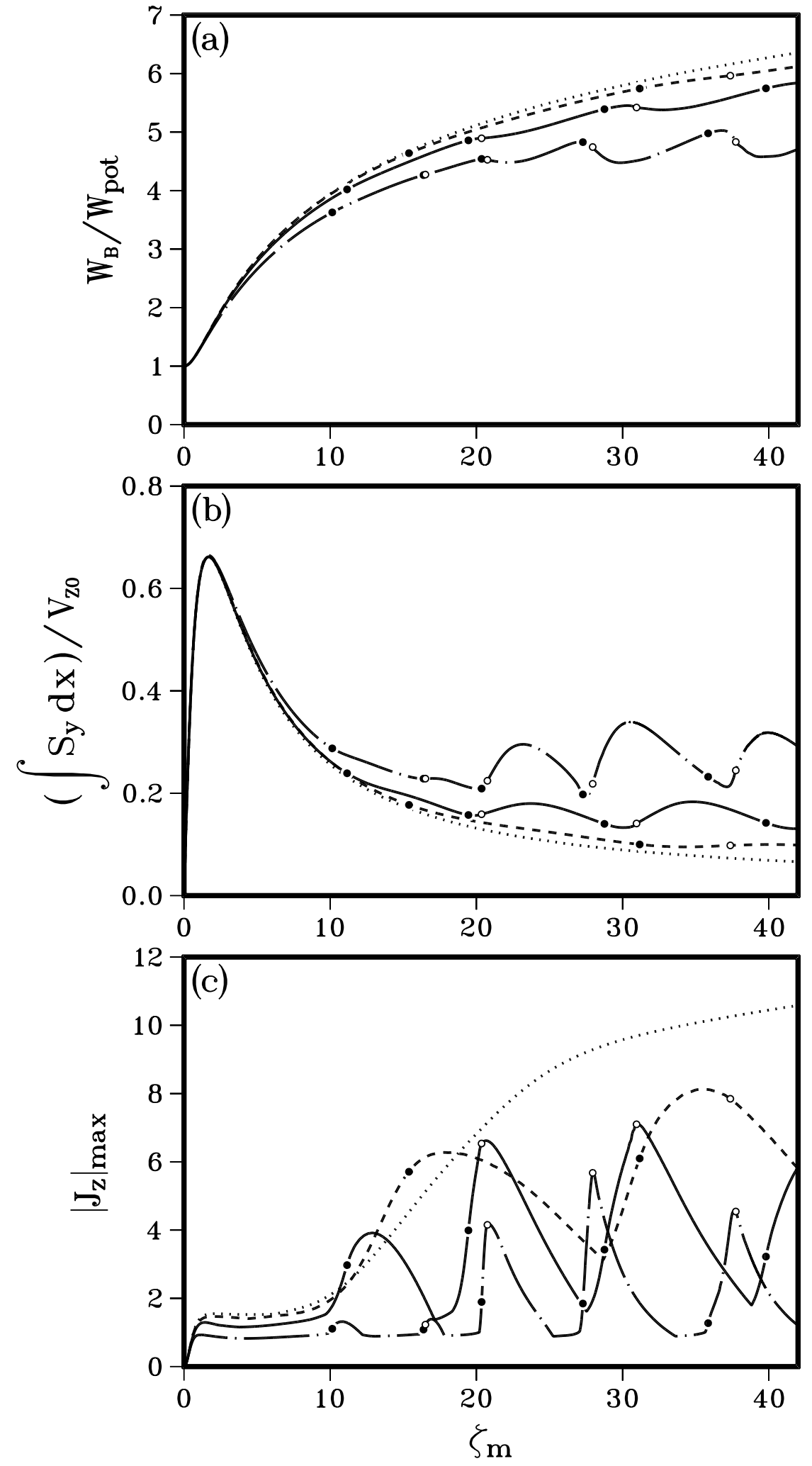
FIG. 3. The evolution of (a) magnetic energy in units of the potential field energy, (b) Poynting flux through the bottom boundary divided by $V_{z 0}$, and (c) maximum magnitude of the current density in the current layer under the magnetic island. All the quantities are plotted as a function of $\zeta_{m}$ which is the plasma displacement at $x= \pm L_{0}$. In all three figures, the solid lines represent the case with $\eta=10^{-5}$, the chain-dotted lines represent the case with $\eta=5 \times 10^{-5}$, and the dashed lines represent the case with $\eta=2 \times 10^{-6}$. The dotted lines are for the ideal MHD case $(\eta=0)$. The filled circles denote the formation of a new island in the underlying arcade and the blank circles indicate the completion of the island merging. 

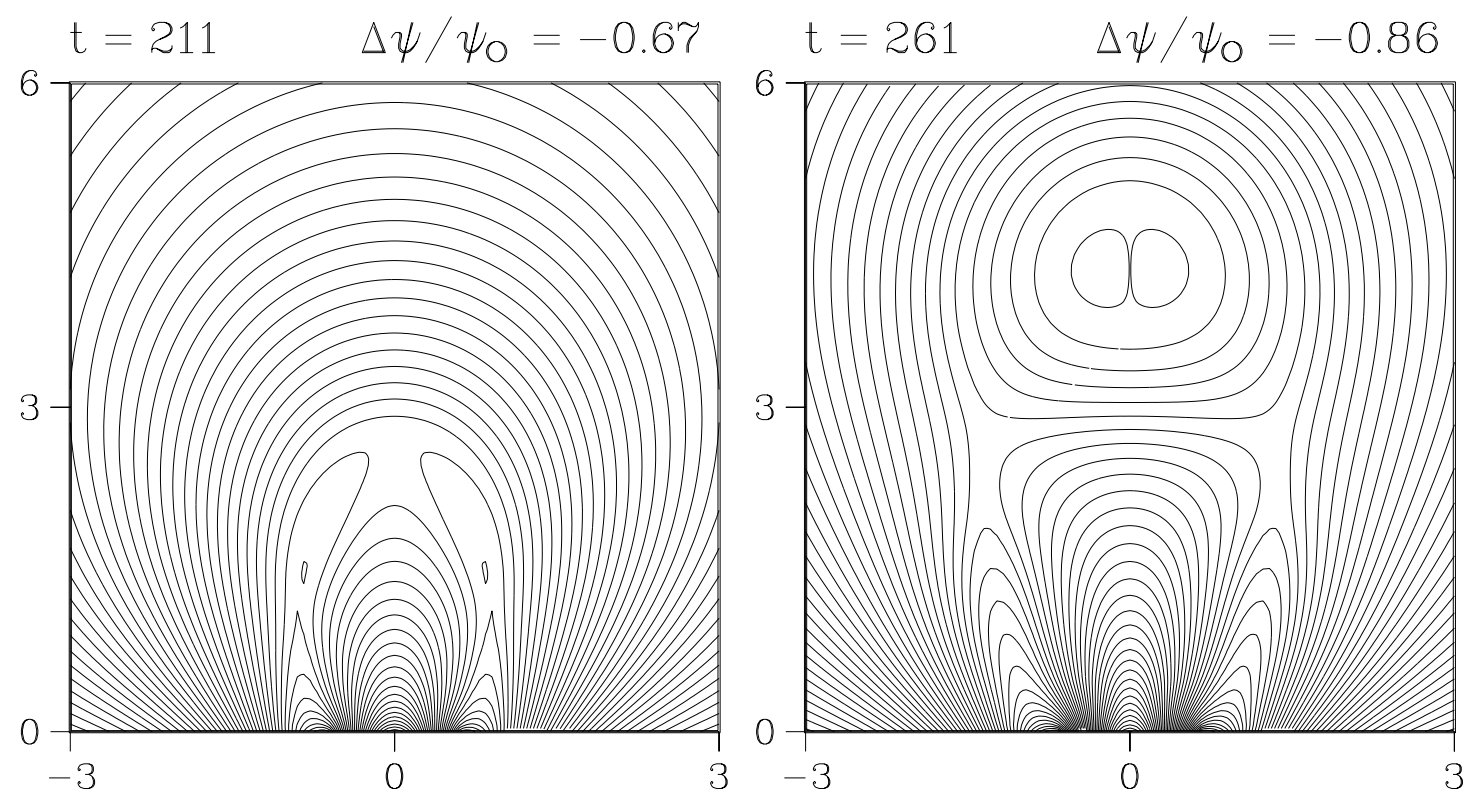

FIG. 4. Formation of magnetic islands by magnetic reconnection between the pre-existing field and the emerging field both having parallel axial field components. Small magnetic islands eventually coalesce to form one big island. The emerging flux is denoted by $\Delta \psi$. 


\section{External Distribution}

Plasma Research Laboratory, Australian National University, Australia

Professor I.R. J ones, Flinders University, Australia

Professor J oão Canalle, Instituto de Fisica DEQ/IF - UERJ , Brazil

Mr. Gerson O. Ludwig, Instituto Nacional de Pesquisas, Brazil

Dr. P.H. Sakanaka, Instituto Fisica, Brazil

The Librarian, Culham Laboratory, England

Library, R61, Rutherford Appleton Laboratory, England

Mrs. S.A. Hutchinson, JET Library, England

Professor M.N. Bussac, Ecole Polytechnique, France

Librarian, Max-Planck-Institut für Plasmaphysik, Germany

J olan Moldvai, Reports Library, MTA KFKI-ATKI, Hungary

Dr. P. Kaw, Institute for Plasma Research, India

Ms. P.J . Pathak, Librarian, Insitute for Plasma Research, India

Ms. Clelia De Palo, Associazione EURATOM-ENEA, I taly

Dr. G. Grosso, Instituto di Fisica del Plasma, Italy

Librarian, Naka Fusion Research Establishment, J AERI, J apan

Library, Plasma Physics Laboratory, Kyoto University, J apan

Research Information Center, National Institute for Fusion Science, J apan

Dr. O. Mitarai, Kyushu Tokai University, J apan

Library, Academia Sinica, Institute of Plasma Physics, People's Republic of China

Shih-Tung Tsai, Institute of Physics, Chinese Academy of Sciences, People's Republic of China

Dr. S. Mirnov, TRINITI, Troitsk, Russian Federation, Russia

Dr. V.S. Strelkov, Kurchatov Institute, Russian Federation, Russia

Professor Peter Lukac, Katedra Fyziky Plazmy MFF UK, Mlynska dolina F-2, Komenskeho Univerzita, SK-842 15 Bratislava, Slovakia

Dr. G.S. Lee, Korea Basic Science Institute, South Korea

Mr. Dennis Bruggink, Fusion Library, University of Wisconsin, USA

Institute for Plasma Research, University of Maryland, USA

Librarian, Fusion Energy Division, Oak Ridge National Laboratory, USA

Librarian, Institute of Fusion Studies, University of Texas, USA

Librarian, Magnetic Fusion Program, Lawrence Livermore National Laboratory, USA

Library, General Atomics, USA

Plasma Physics Group, Fusion Energy Research Program, University of California at San Diego, USA

Plasma Physics Library, Columbia University, USA

Alkesh Punjabi, Center for Fusion Research and Training, Hampton University, USA

Dr. W.M. Stacey, Fusion Research Center, Georgia Institute of Technology, USA

Dr. J ohn Willis, U.S. Department of Energy, Office of Fusion Energy Sciences, USA

Mr. Paul H. Wright, Indianapolis, Indiana, USA 
The Princeton Plasma Physics Laboratory is operated by Princeton University under contract with the U.S. Department of Energy.

\author{
Information Services \\ Princeton Plasma Physics Laboratory \\ P.O. Box 451 \\ Princeton, NJ 08543
}

Phone: 609-243-2750

Fax: 609-243-2751

e-mail: pppl_info@pppl.gov

Internet Address: http://www.pppl.gov 